\title{
Proof of Ito's Formula for Ito's Process in Nonstandard Analysis
}

\author{
Shuya Kanagawa ${ }^{1}$, Kiyoyuki Tchizawa ${ }^{2}$ \\ ${ }^{1}$ Department of Mathematics, Tokyo City University Setagaya, Tokyo, Japan \\ ${ }^{2}$ Institute of Administration Engineering, Ltd., Tokyo, Japan \\ Email: skanagaw@tcu.ac.jp, tchizawa@kthree.co.jp
}

How to cite this paper: Kanagawa, S. and Tchizawa, K. (2019) Proof of Ito's Formula for Ito's Process in Nonstandard Analysis. Applied Mathematics, 10, 561-567. https://doi.org/10.4236/am.2019.107039

Received: June 17, 2019

Accepted: July 19, 2019

Published: July 22, 2019

Copyright (c) 2019 by author(s) and Scientific Research Publishing Inc. This work is licensed under the Creative Commons Attribution International License (CC BY 4.0).

http://creativecommons.org/licenses/by/4.0/ (c) (i) Open Access

\begin{abstract}
In our previous paper [1], we proposed a non-standardization of the concept of convolution in order to construct an extended Wiener measure using nonstandard analysis by E. Nelson [2]. In this paper, we consider Ito's integral with respect to the extended Wiener measure and extend Ito's formula for Ito's process. Because of doing the extension of Ito's formula, we could treat stochastic differential equations in the sense of nonstandard analysis. In this framework, we need the nonstandardization of convolution again. It was not yet proved in the last paper, therefore we shall provide the proof.
\end{abstract}

\section{Keywords}

Ito's Process, Stochastic Differential Equation, S-Continuity, Nonstandard Analysis

\section{Introduction}

As for an analysis of stochastic differential equations driven by extended Wiener process in the sense of nonstandard analysis, we need to extend "Ito's formula" for Wiener process or Ito's process. In the previous paper, we extended a concept of convolution in Fourier series to the case of nonstandard analysis. According to the result, we shall extend some theorems in probability theory, for example, the law of large numbers and the central limit theorem, and shall reconstruct Ito's formula by using nonstandard analysis. We shall give the proof of the reconstruction of Ito's formula in the case that the convolution of probability density which functions in a nonstandard extension is convergent for some functional $F(t, X(t))$ of Ito's process $X(t)$. The problem was not solved still now.

If the convolution is not convergent, what kind of problem does it occur? In 
Taylor expansion of $F(t, X)$, the higher terms may not vanish. Then, Ito's formula does not be established. As to what we shall give extended law of large numbers and extended central limit theorem, they will be provided precisely in the next paper.

\section{Ito's Integral for Extended Wiener Process in Nonstandard}

In our previous paper [1], we showed that Fourier series can be described by the convolution in nonstandard analysis, then the series of i.i.d. random variables using Loeb measure [3] converges in $L^{2}$ sense under some moment condition. Therefore, the definition of stochastic integral in classical probability theory can be extended by the way of nonstandard analysis [4], [5].

Furthermore, we need to prove some laws of large numbers for i.i.d. random variables to show the convergence to a stochastic integral.

In fact, we use an extended concept of the convolution to investigate the expectation or the distribution of series of i.i.d. random variables for the nonstandardization of the law of large numbers.

In order to prove the convergence of sums of higher order of $\Delta W_{k}$ such as $\left(\Delta W_{k}\right)^{3},\left(\Delta W_{k}\right)^{5}, \cdots$ in the proof of Ito formula, we need to extend the law of large numbers for $\Delta W_{k}$ in the sense of nonstandard.

From the above discussion, we shall define the stochastic integral in nonstandard analysis.

Let $\Delta t$ be the infinitesimal and $N=\frac{T}{\Delta t}$. The extended Winer process is defined as follows.

Definition 2.1. Let $N_{t}=\frac{t}{\Delta t}, 0 \leq t \leq T$ and $N=N_{T}$. Assume that a sequence of i.i.d. random variables $\left\{\Delta W_{k}, k=1, \cdots, N\right\}$ has the distribution

$$
P\left\{\Delta W_{k}=\sqrt{\Delta t}\right\}=P\left\{\Delta W_{k}=-\sqrt{\Delta t}\right\}=\frac{1}{2}
$$

for each $k=1, \cdots, N$. An extended Wiener process $\{W(t), t \geq 0\}$ is defined by

$$
W(t)=\sum_{k=1}^{N_{t}} \Delta W_{k}, 0 \leq t \leq T .
$$

Ito's integral (stochastic integral) in the nonstandard sense is defined as follows.

Definition 2.2. Let $\{W(t), 0 \leq t \leq T\}$ be an extended Wiener process. Assume that an adapted process $\{\sigma(t), 0 \leq t \leq T\}$ with respect the Wiener process $W(t)$ is defined by

$$
\sigma(t)=Y_{k}, t_{k} \leq t<t_{k+1}, k=0,1, \cdots, N,
$$

where $t_{k}=k \Delta t, k=0,1, \cdots, N$ and each $Y_{k}$ is measurable with respect to $\left\{W(t), 0 \leq t \leq t_{k}\right\}$. Assume that

$$
E\left[Y_{k}^{2}\right]<\infty, k \geq 1 .
$$


A stochastic integral in nonstandard analysis is defined by

$$
\int_{0}^{T} \sigma(t) \mathrm{d} W(t)=\sum_{k=1}^{N} Y_{k-1} \Delta W_{k},
$$

where $Y_{0}$ is independent of $\{W(t), t \geq 0\} \quad\left\{\Delta W_{k}, k=1, \cdots, N\right\}$ is a sequence of i.i.d. random variables with the distribution

$$
P\left\{\Delta W_{k}=\sqrt{\Delta t}\right\}=P\left\{\Delta W_{k}=-\sqrt{\Delta t}\right\}=\frac{1}{2} .
$$

Remark 1. In classical (standard) probability theory, Ito's integral $\int_{0}^{T} \sigma(t) \mathrm{d} W(t)$ is well defined under the condition of the existence of the variance of $\sigma(t)$ for each $0 \leq t \leq T$. In nonstandard analysis, the convergence of the series in (5) may not be ensured. On the other hand, take note that we have already given some sufficient conditions for the convergence of the convolution in Fourier series. See [1].

\section{Proof of Ito's Formula for Extended Wiener Process in Nonstandard}

From the concept of Ito's integral for the extended Wiener process, we provide Ito's formula for the extended Wiener process.

Theorem 3.1. Let $F(t, x)$ be of $C^{3}$. Assume that the condition (4) is satisfied, then we have the following for the extended Wiener Process $W(t)$. For any $T \geq 0$,

$$
\begin{aligned}
F(t, W(T))= & F(0, W(0))+\int_{0}^{T} F_{t}(t, W(t)) \mathrm{d} t \\
& +\int_{0}^{T} F_{x}(t, W(t)) \mathrm{d} W(t)+\frac{1}{2} \int_{0}^{T} F_{x x}(t, W(t)) \mathrm{d} t .
\end{aligned}
$$

Proof.

$$
\begin{aligned}
F & (t+\Delta t, W(t+\Delta t))-F(t, W(t)) \\
= & F_{t}(t, W(t)) \Delta t+F_{x}(t, W(t))\{W(t+\Delta t)-W(t)\} \\
& +\frac{1}{2} F_{t t}(t, W(t))(\Delta t)^{2}+F_{t x}(t, W(t)) \Delta t\{W(t+\Delta t)-W(t)\} \\
& +\frac{1}{2} F_{x x}(t, W(t))\{W(t+\Delta t)-W(t)\}^{2}+\frac{1}{3 !} F_{t t t}(t, W(t))(\Delta t)^{3} \\
+ & \frac{3}{3 !} F_{t t x}(t, W(t))(\Delta t)^{2}\{W(t+\Delta t)-W(t)\} \\
& +\frac{3}{3 !} F_{t x x}(t, W(t)) \Delta t\{W(t+\Delta t)-W(t)\}^{2} \\
& +\frac{1}{3 !} F_{x x x}(t, W(t))\{W(t+\Delta t)-W(t)\}^{3}+\cdots
\end{aligned}
$$

Put

$$
\begin{gathered}
N=\left[\frac{T}{\Delta t}\right], t_{k}=k \Delta t, k=0,1, \cdots, N-1, \\
\Delta W_{k}=W\left(t_{k}\right)-W\left(t_{k-1}\right), k=0,1, \cdots, N-1
\end{gathered}
$$


and

$$
\Delta W_{N}=W(T)-W\left(t_{N-1}\right)
$$

then,

$$
\begin{aligned}
F & (t, W(T))-F(0, W(0)) \\
= & \sum_{k=1}^{N} F_{t}\left(t_{k-1}, W\left(t_{k-1}\right)\right) \Delta t+\sum_{k=1}^{N} F_{x}\left(t_{k-1}, W\left(t_{k-1}\right)\right) \Delta W_{k} \\
& +\frac{1}{2} \sum_{k=1}^{N} F_{t t}\left(t_{k-1}, W\left(t_{k-1}\right)\right)(\Delta t)^{2}+\sum_{k=1}^{N} F_{t x}\left(t_{k-1}, W\left(t_{k-1}\right)\right) \Delta t \Delta W_{k} \\
& +\frac{1}{2} \sum_{k=1}^{N} F_{x x}\left(t_{k-1}, W\left(t_{k-1}\right)\right) \Delta t+\frac{1}{3 !} \sum_{k=1}^{N} F_{t t t}\left(t_{k-1}, W\left(t_{k-1}\right)\right)(\Delta t)^{3} \\
& +\frac{3}{3 !} \sum_{k=1}^{N} F_{t t x}\left(t_{k}, W\left(t_{k}\right)\right)(\Delta t)^{2} \Delta W_{k}+\frac{3}{3 !} \sum_{k=1}^{N} F_{t x x}\left(t_{k}, W\left(t_{k}\right)\right)(\Delta t)^{2} \\
& +\frac{1}{3 !} \sum_{k=1}^{N} F_{x x x}\left(t_{k}, W\left(t_{k}\right)\right) \Delta t \Delta W_{k}+\cdots \\
= & \int_{0}^{T} F_{t}(t, W(t)) \mathrm{d} t+\int_{0}^{T} F_{x}(t, W(t)) \mathrm{d} W(t) \\
& +\frac{1}{2}\left\{\int_{0}^{T} F_{t t}(t, W(t)) \mathrm{d} t\right\} \Delta t+\left\{\int_{0}^{T} F_{t x}(t, W(t)) \mathrm{d} W(t)\right\} \Delta t \\
& +\frac{1}{2} \int_{0}^{T} F_{x x}(t, W(t)) \mathrm{d} t+\frac{1}{3 !}\left(\Delta t^{2}\right)\left\{\int_{0}^{T} F_{t t t}(t, W(t)) \mathrm{d} t\right\} \\
& +\frac{3}{3 !} \Delta t\left\{\int_{0}^{T} F_{t t x}(t, W(t)) \mathrm{d} W(t)\right\}+\frac{3}{3 !} \Delta t\left\{\int_{0}^{T} F_{t x x}(t, W(t)) \mathrm{d} t\right\} \\
& +\frac{1}{3 !} \Delta t\left\{\int_{0}^{T} F_{x x x}(t, W(t)) \mathrm{d} W(t)\right\}+\cdots \\
= & \int_{0}^{T} F_{t}(t, W(t)) \mathrm{d} t+\int_{0}^{T} F_{x}(t, W(t)) \mathrm{d} W(t) \\
& +\frac{1}{2} \int_{0}^{T} F_{x x}(t, W(t)) \mathrm{d} t
\end{aligned}
$$

\section{Proof of Ito's Formula for Ito's Process in Nonstandard}

Let $X(t)$ be Ito's process defined by

$$
X(t)=X_{0}+\int_{0}^{t} b(s) \mathrm{d} s+\int_{0}^{t} \sigma(s) \mathrm{d} W(s), 0 \leq t \leq T
$$

where $b(s)$ and $\sigma(s)$ are adapted processes with respect to a Wiener process $W(t)$. Then, we have Ito's formula for the Ito's process.

Theorem 4.1. Let $F(t, x)$ be of $C^{3}$, then we have the following. For any $T \geq 0$,

$$
\begin{aligned}
F(t, X(T))= & F(0, X(0))+\int_{0}^{T} F_{t}(t, X(t)) \mathrm{d} t \\
& +\int_{0}^{T} F_{x}(t, X(t)) b(t) \mathrm{d} t+\int_{0}^{T} F_{x}(t, X(t)) \sigma(t) \mathrm{d} W(t) \\
& +\frac{1}{2} \int_{0}^{T} F_{x x}(t, X(t)) \sigma(t)^{2} \mathrm{~d} t .
\end{aligned}
$$

Proof. We provide the proof by using nonstandard analysis.

From the Taylor expansion for the two-dimensional function $F(t, X(t))$, we 
have the following.

$$
\begin{aligned}
F & (t+\Delta t, X(t+\Delta t))-F(t, X(t)) \\
= & F_{t}(t, X(t)) \Delta t+F_{x}(t, X(t))\{X(t+\Delta t)-X(t)\} \\
& +\frac{1}{2} F_{t t}(t, X(t))(\Delta t)^{2}+F_{t x}(t, X(t)) \Delta t\{X(t+\Delta t)-X(t)\} \\
& +\frac{1}{2} F_{x x}(t, X(t))\{X(t+\Delta t)-X(t)\}^{2}+\frac{1}{3 !} F_{t t t}(t, X(t))(\Delta t)^{3} \\
& +\frac{3}{3 !} F_{t t x}(t, X(t))(\Delta t)^{2}\{X(t+\Delta t)-X(t)\} \\
& +\frac{3}{3 !} F_{t x x}(t, X(t)) \Delta t\{X(t+\Delta t)-X(t)\}^{2} \\
& +\frac{1}{3 !} F_{x x x}(t, X(t))\{X(t+\Delta t)-X(t)\}^{3}+\cdots
\end{aligned}
$$

In nonstandard analysis, we can represent the Ito's process $X(t)$ for the extended Wiener process by

$$
\begin{aligned}
X(t) & =X_{0}+\int_{0}^{t} b(s) \mathrm{d} s+\int_{0}^{t} \sigma(s) \mathrm{d} W(s) \\
& =X_{0}+\sum_{k=1}^{N} b\left(t_{k-1}\right) \Delta t+\sum_{k=1}^{N} \sigma\left(t_{k-1}\right) \Delta W_{k}, \quad 0 \leq t \leq T,
\end{aligned}
$$

where for infinitesimal $\Delta t$

$$
N=\left[\frac{T}{\Delta t}\right], t_{k}=k \Delta t, k=0,1, \cdots, N
$$

and

$$
\Delta W_{k}=W\left(t_{k}\right)-W\left(t_{k-1}\right), \quad k=0,1, \cdots, N .
$$

Therefore, the difference of $X(t)$ can be represented by the following,

$$
\Delta X_{k}=X\left(t_{k}\right)-X\left(t_{k-1}\right)=b\left(t_{k-1}\right) \Delta t+\sigma\left(t_{k-1}\right) \Delta W_{k} .
$$

On the other hand,

$$
\left(\Delta W_{k}\right)^{2 n}=(\Delta t)^{n} \text { a.s. }
$$

for each $n \geq 1$ from (1).

Thus, we have the following.

$$
\begin{aligned}
F & (t, X(T))-F(0, X(0)) \\
= & \sum_{k=1}^{N} F_{t}\left(t_{k-1}, X\left(t_{k-1}\right)\right) \Delta t+\sum_{k=1}^{N} F_{x}\left(t_{k-1}, X\left(t_{k-1}\right)\right) \Delta X_{k} \\
& +\frac{1}{2} \sum_{k=1}^{N} F_{t t}\left(t_{k-1}, X\left(t_{k-1}\right)\right)(\Delta t)^{2}+\sum_{k=1}^{N} F_{t x}\left(t_{k-1}, X\left(t_{k-1}\right)\right) \Delta t X W_{k} \\
& +\frac{1}{2} \sum_{k=1}^{N} F_{x x}\left(t_{k-1}, X\left(t_{k-1}\right)\right)\left\{\Delta X_{k}\right\}^{2}+\frac{1}{3 !} \sum_{k=1}^{N} F_{t t t}\left(t_{k-1}, X\left(t_{k-1}\right)\right)(\Delta t)^{3} \\
& +\frac{3}{3 !} \sum_{k=1}^{N} F_{t t x}\left(t_{k}, X\left(t_{k}\right)\right)(\Delta t)^{2} \Delta X_{k}+\frac{3}{3 !} \sum_{k=1}^{N} F_{t x x}\left(t_{k}, X\left(t_{k}\right)\right) \Delta t\left(\Delta X_{k}\right)^{2} \\
& +\frac{1}{3 !} \sum_{k=1}^{N} F_{x x x}\left(t_{k}, X\left(t_{k}\right)\right)\left(\Delta X_{k}\right)^{3}+\cdots \\
= & \sum_{k=1}^{N} F_{t}\left(t_{k-1}, X\left(t_{k-1}\right)\right) \Delta t
\end{aligned}
$$




$$
\begin{aligned}
& +\sum_{k=1}^{N} F_{x}\left(t_{k-1}, X\left(t_{k-1}\right)\right)\left\{b\left(t_{k-1}\right) \Delta t+\sigma\left(t_{k-1}\right) \Delta W_{k}\right\} \\
& +\frac{1}{2} \sum_{k=1}^{N} F_{t t}\left(t_{k-1}, X\left(t_{k-1}\right)\right)(\Delta t)^{2} \\
& +\sum_{k=1}^{N} F_{t x}\left(t_{k-1}, X\left(t_{k-1}\right)\right) \Delta t\left\{b\left(t_{k-1}\right) \Delta t+\sigma\left(t_{k-1}\right) \Delta W_{k}\right\} \\
& +\frac{1}{2} \sum_{k=1}^{N} F_{x x}\left(t_{k-1}, X\left(t_{k-1}\right)\right)\left\{b\left(t_{k-1}\right) \Delta t+\sigma\left(t_{k-1}\right) \Delta W_{k}\right\}^{2} \\
& +\frac{1}{3 !} \sum_{k=1}^{N} F_{t t t}\left(t_{k-1}, X\left(t_{k-1}\right)\right)(\Delta t)^{3} \\
& +\frac{3}{3 !} \sum_{k=1}^{N} F_{t t x}\left(t_{k}, X\left(t_{k}\right)\right)(\Delta t)^{2}\left\{b\left(t_{k-1}\right) \Delta t+\sigma\left(t_{k-1}\right) \Delta W_{k}\right\} \\
& +\frac{3}{3 !} \sum_{k=1}^{N} F_{t x x}\left(t_{k}, X\left(t_{k}\right)\right) \Delta t\left\{b\left(t_{k-1}\right) \Delta t+\sigma\left(t_{k-1}\right) \Delta W_{k}\right\}^{2} \\
& +\frac{1}{3 !} \sum_{k=1}^{N} F_{x x x}\left(t_{k}, X\left(t_{k}\right)\right)\left\{b\left(t_{k-1}\right) \Delta t+\sigma\left(t_{k-1}\right) \Delta W_{k}\right\}^{3}+\cdots \\
& =\int_{0}^{T} F_{t}(t, X(t)) \mathrm{d} t+\int_{0}^{T} F_{x}(t, X(t)) b(t) \mathrm{d} t \\
& +\int_{0}^{T} F_{x}(t, X(t)) \sigma(t) \mathrm{d} W(t)+\frac{1}{2} \int_{0}^{T} F_{x x}(t, X(t)) \sigma(t)^{2} \mathrm{~d} t
\end{aligned}
$$

Thus we prove Ito's formula for the extended Ito's process.

Remark 2. Let $X_{1}, X_{2}, \cdots, X_{n}$ be independent random variables with density functions $f_{1}, f_{2}, \cdots, f_{n}$, respectively. Then, the distribution of $\sum_{k=1}^{n} X_{k}$ can be represented by convolution $f_{1} * f_{2} * \cdots * f_{n}$. In standard analysis, for the Fourier transform of the convolution

$$
\mathcal{F}\left(f_{1} * f_{2} * \cdots * f_{n}\right)=\prod_{k=1}^{n} \mathcal{F}\left(f_{k}\right)
$$

is established, where $\mathcal{F}\left(f_{k}\right)$ is the Fourier transform of $f_{k}$.

From our previous paper [1], the result can be extended in the sense of nonstandard. See pp.976. Therefore, it is applied for the extension of limit theorems as like central limit theorem, law of large numbers and so on.

\section{Conclusions}

In classical (standard) probability theory, the stochastic integral

$$
\int_{0}^{T} \sigma(t) \mathrm{d} W(t)
$$

is defined under the condition of the existence of the variance of $\sigma(t)$ for each $0 \leq t \leq T$. In nonstandard analysis, the convergence of the series in (5) is proved from the above arguments. On the proof of Ito's formula, it can be applied for other estimations as the same way.

Furthermore, the proof of Ito's formula in nonstandard analysis becomes simple rather than the proof in standard one. 


\section{Supported}

The first author is supported in part by Grant-in-Aid Scientific Research (C), No.18K03431, Ministry of Education, Science and Culture, Japan.

\section{Conflicts of Interest}

The authors declare no conflicts of interest regarding the publication of this paper.

\section{References}

[1] Kanagawa, S., Nishiyama, R. and Tchizawa, K. (2018) Extended Wiener Measure by Nonstandard Analysis for Financial Time Series. Applied Mathematics, 9, 975-984. https://doi.org/10.4236/am.2018.98066

[2] Nelson, E. (1977) Internal Set Theory: A New Approach to Nonstandard Analysis. Bulletin of the American Mathematical Society, 83, 1165-1198. https://doi.org/10.1090/S0002-9904-1977-14398-X

[3] Hurd, A.E. and Loeb, P.A. (1985) Introduction to Nonstandard Real Analysis. Acad. Press, New York.

[4] Benot, E. (1989) Diffusions discrètes et mécanique stochastique. Prépubli. Lab. Math. J. Dieudonné, Université de Nice.

[5] Benot, E. (1995) Random Walks and Stochastic Differential Equations. In: Diener, F. and Diener, M., Eds., Nonstandard Analysis in Practice, Springer, Berlin, Heidelberg, 71-90. https://doi.org/10.1007/978-3-642-57758-1_4 